\title{
Comparison of 2D versus 3D diffusion analysis at Nanowire Electrodes: Finite element analysis and experimental study
}

\author{
Benjamin O’Sullivan ${ }^{\mathrm{a}}$, Shane O’Sullivan ${ }^{\mathrm{a}}$, Tarun Naruyan ${ }^{\mathrm{a}}$, Han Shao ${ }^{\mathrm{a}}$, Bernardo Patella ${ }^{\mathrm{ab}}$, Ian Seymour , \\ Rosalinda Inguanta ${ }^{\mathrm{b}}$, Alan O’Riordan ${ }^{\mathrm{a}}$ \\ a Nanotechnology Group, Tyndall National Institute, University College Cork, Cork T12 R5CP, Ireland \\ b Laboratorio di Chimica Fisica Applicata, Dipartimento di Ingegneria, Università of Palermo, Viale delle Scienze, Palermo, \\ Italy
}

Corresponding Author: alan.oriordan@tyndall.ie

Supporting Information is provide at the end of the manuscript

\begin{abstract}
In electroanalysis, finite element simulations of electrochemical processes occurring at electrodes are used to provide key insight into experimental design in relation to diffusion profiles and expected currents. The diffusion domain approach (DDA) offers a means of reducing a three dimensional design to two dimensions to ease computational demands. However, the DDA approach can be limited when basic assumptions, for example that all electrodes in an array are equivalent, are incorrect. Consequently, to get a more realistic view of molecular diffusion to nanoelectrodes, it is necessary to undertake simulations in 3D. In this work, two and three dimensional models of electrodes comprising of (i) single nanowires, (ii) arrays of nanowires and (iii) interdigitated arrays of nanowires operating in generator-collector mode, are undertaken and compared to experimental results obtained from fabricated devices. The 3D simulations predict a higher extracted current for a single nanowires and diffusionally independent nanowire arrays when compared to 2D simulations since, unlike the 2D model, they take into account molecular diffusion to and from the nanowire termini. This current difference was observed to increase with increasing electrode width and decrease with electrode length. When the nanowire arrays were diffusionally overlapped, they behaved as an electrode of larger width, and the divergence between the two models increased. By contrast, in generator-collector mode, using interdigitated nanowire arrays, the difference between extracted current values obtained using two models was significantly lower. Simulations indicated however that a higher collection efficiency was predicted by the 2D model when compared to the 3D model. Electrochemical experiments were undertaken to confirm the simulation study and demonstrated that
\end{abstract}


the extracted currents from 3D simulations more closely mapped onto experimentally measured currents.

\section{Keywords}

Finite element analysis simulation; diffusion domain approach; 3D modelling;

Electroanalysis; Nanowire interdigitated electrode array. (5 max)

\section{Introduction}

The prevalence of electrochemical sensors at the ultramicro- and nano-scale has become more common in recent years due to advances in nanofabrication techniques and, as such, they are now being used for the detection of a wide range of analytes. [1-5]

Ultramicroelectrodes offer enhanced performance due to time independent analyte masstransport (radial diffusion) occurring to these electrodes when compared to larger electrodes. As such they have been applied to a variety of different electrochemical techniques including voltammetry, amperometric and generator - collector approaches. [6-9] In addition, they are often fabricated in array configurations of high density to provide even higher sensitivity. In scanning voltammetry approaches, when nanoelectrodes in an array are diffusionally independent, the measured current increases linearly with the number of electrodes in the array. [10] However, when the electrodes within these arrays are closely spaced, i.e., not diffusionally independent, analyte diffusional profiles at the individual nanostructures overlap, a time dependent (planar diffusion) response is observed and the array behaves as a single larger electrode; of a size equivalent to the area of the array. [11] The resultant current responses do not increase lineally with the number of electrodes in the array and typically yields a current response similar to that of the larger electrode. [12] By contrast, closely space electrodes are required for generator-collector based electrochemistry in order to establish redox cycling, which is a well-established method of enhancing the current response of an electrode array. In this approach two working (or arrays of) electrodes, a generator and a collector, are biased at different potentials. These potentials are typically selected to be at the oxidation and reduction potentials of the molecule of interest, which diffuses or cycles between the electrodes undergoing redox reactions at each. When this occurs, the molecule is said to redox cycle between the electrodes, which can yield a significantly increase in measured current arising due to a number of electronic transfer events per molecule. The technique has been employed at a variety of electrode geometries, including rotating ring disk[13], recessed nanoring-ring microarrays [14], and interdigitated bands[15]. 
The mass transport to an electrode surface has been widely studied $[16,17]$ in the literature where it has been reported that radial diffusion profiles become dominant when the electrode critical dimension shrinks below the thickness of the diffusion layer. Consequently, when designing sensors to address specific deployment use cases it is imperative to understand the impact of design architecture on sensor performance. Simulations not only provide an $a$ priori guide to sensor fabrication, but can also serve to enable a subsequent comparison for experimental results as part of the characterisation/evaluation process. While modelling a system in $3 \mathrm{D}$ is a realistic possibility, the approach is significantly more demanding in terms of computer power, than 2D simulations, and simulations often take days to resolve. To this end, the diffusion domain approach (DDA) has been used to convert 3D architectural layouts to $2 \mathrm{D}$ to greatly reduce computational demands. [18] The DDA can be applied most successfully to symmetrical electrodes and electrode arrays. [19] For instance, Xiong et al. modelled ion transport in nanogap electrochemical cells [20] while Ma et al. studied redox cycling in nanopore electrode arrays. [21] Similarly, 2D modelling of generator-collector systems has successfully demonstrated the relationship between interelectrode spacing and collection efficiency. [22]

The DDA, while elegant, remains an approximation, and, is thus limited. Godino et al. showed the limits of the DDA for nanodisk arrays, where assumptions that "electrodes across the array were equivalent" no longer held true due to enhanced radial diffusion based mass transport occurring at the edges when compared to electrodes within the arrays. [11] The limits of the DDA approach for micro and nanobands of finite length was investigated by Strutwolf [23]. The results of that work showed significant errors arose between the 2D and 3D models when the length of the electrode approached its width. Cutress et al modelled the chronoamperometric transient for microband electrodes with a 3D approach, which enabled the incorporation of the increased contributions of short edge diffusion at finite electrode lengths into the analysis [24]. Application of 3D FEM analysis using commercial software had been limited due to computational power required to provide high level meshing at smaller length scales [25]. However, as computing power has improved, 3D simulations have become more widely reported in the literature. Woodvine et al. used 3D FEM simulations of their square electrodes to show enhanced diffusion occurring at micro-square corners[26]. Molina et al. employed a 3D model for their microdisk in a microfluidic channel to extract a limiting current and obtain information on structure imperfections. [27] Atighilorestani et al. utilised a 3D model for their array of nanorings, due to a 2D model being unable to account 
for the differing diffusion profiles of electrodes at the edge of the array versus those at the centre of the array. [28] The recent simulation work of Britz et al, for rectangular electrodes [29], and Batchelor-McAuley et al, showed diffusion to a 3D cube [30]. These simulations highlighted the non-uniform flux to a surface at the edge and corner discontinuities.

Concerning 1D nanoband structures, in recent years our group and others have designed, fabricated and/or integrated nanowires (with a lengths of $45 \mu \mathrm{m}$ or smaller) into functional devices. [31-34] With these devices, it has been observed that the DDA approximations can deviate significantly from experimental results which can lead to a large source of error when trying to extract electrochemical currents; required to optimise sensor design. In this work, we endeavour to elucidate this source of error by undertaking a simulation study of on-chip nanowire based electrochemical devices, with a range of different dimensions, employing both 2D and 3D model approaches. Using standard nanoelectronic fabrication techniques we also fabricate a number of the devices we simulated including: single-, arrays- and interdigitatednanowire electrode devices and undertake electrochemical experiments to benchmark and validate the developed models. We demonstrate how analyte diffusion to and from the termini of the nanowires, which is not taken into account using 2D approaches, can significant impact on simulated currents extracted using the electroanalytical Butler-Volmer equation for both cyclic voltammetry and generator - collector detection approaches at these devices.

\section{Methods}

\subsection{Materials, Electrode Fabrication and Characterization}

Ferrocene carboxylic acid $(\mathrm{FcCOOH})$ and phosphate buffer saline (PBS) tablets were purchased from Sigma Aldrich, Ireland and used as received. All solutions were prepared

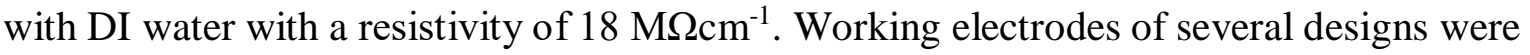
fabricated including: single nanowires with varying widths $100-500 \mathrm{~nm}$, arrays of 3 nanowires with $100 \mathrm{~nm}$ width and separated by $15 \mu \mathrm{m}$, and interdigitated nanowire electrodes with $100 \mathrm{~nm}$ width and varying intercomb separation of $200-1000 \mathrm{~nm}$. All electrodes were fabricated with $60 \mathrm{~nm}$ height $(10 / 50 \mathrm{~nm} \mathrm{Ti} / \mathrm{Au})$ and were $45 \mu \mathrm{m}$ in length (as defined by the opening in the passivation window). Silicon chip sensor devices also consisted of an SD pinout, gold nanowire working electrodes, a gold counter electrode and a platinum pseudo reference electrode, which were fabricated as described previously. In brief, nanowires were 
patterned in resist by direct beam writing and metal evaporation ( $\mathrm{Ti} / \mathrm{Au} 10 / 50 \mathrm{~nm}$ ) followed by standard lift-off techniques. Optical photolithography, metal evaporation (Ti/Au 10/90), and lift-off procedures were then employed to overlay electrical interconnection tracks including peripheral probe pads. Macroscale gold and platinum counter and pseudo-reference electrodes, respectively, was also deposited during this process. Finally, a silicon nitride passivation layer (500 $\mathrm{nm}$ thick) was deposited to passivate the entire chip and windows selectively opened with a dry etch to allow exclusive contact between the working, reference and counter electrodes with the solution of interest. Electrode width was characterised by means of scanning electron microscopy, using a FEI QUANTA 650 HRSEM. Electrode height was characterised by means of atomic force microscopy, using a Bruker Nanoscope dimension icon atomic force microscope in tapping mode).

A CHI 920 bi-potentiostat was used for the electrochemical measurements. All experiments were undertaken either in (i) a three electrode setup comprising of working electrode (single or nanowire array) an on-chip gold and on-chip platinum pseudo reference electrodes or (ii) a four electrode setup using interdigitated nanowire electrodes (IDEs) where each comb in the IDE was used as an independent working electrode and again an on-chip gold and on-chip platinum pseudo reference electrodes. The single, arrays and IDE working electrodes were electrochemically characterized by cyclic voltammetry (CV) using $1 \mathrm{mM} \mathrm{FcCOOH}$ in $10 \mathrm{mM}$ PBS, pH 7.4 solution. The potential was cycled between $-0.15 \mathrm{~V}$ and $0.45 \mathrm{~V}$ versus an onchip platinum pseudo-reference electrode using a scan rate of $100 \mathrm{mV} / \mathrm{s}$.

\subsection{Simulations}

A simulation model was designed to explore the oxidation and reduction of a redox molecule FcCOOH at the nanowire electrodes, using the FEA software COMSOL Multiphysics 5.3. As in the work by Wahl et al.[10], simulations were based on the heterogeneous single electron oxidation process of defined as:

$$
O+e^{-} \rightleftharpoons R
$$

These simulations were undertaken to investigate the diffusional mass transport and consequent current extracted current obtained at a range of nanowire widths and arrays in two and three dimensions for a solution of $1 \mathrm{mM} \mathrm{FcCOOH}$ in $10 \mathrm{mM}$ PBS (diffusion coefficient of $\left.5.4 \times 10^{-6} \mathrm{~cm}^{2} \mathrm{~s}^{-1}\right)$. Simulations were carried out to mirror experiments in a potential 
window of -0.15 to $0.45 \mathrm{~V}$ vs an on-chip Pt reference at $100 \mathrm{mV} / \mathrm{s}$. In generator-collector mode, the bias applied to second working electrode surface was maintained at $-0.15 \mathrm{~V}$.

\subsubsection{D Model}

As previously mentioned [32], the DDA was employed to provide a simplified 2D model of the $\mathrm{FcCOOH}$ redox reaction. In this approach, the nanowires were reduced to $50 \mathrm{x} 100 \mathrm{~nm}$ metal rectangles in the middle of a far larger boundary, representing the solution domain. The domain was set to be large enough that the reaction of interest at the centre of the domain was unaffected by the bulk conditions at the domain edge. Diffusion to the nanowires was modelled by Fick's $2^{\text {nd }}$ Law. Migration and convection were assumed to be negligible due to excess supporting electrolyte and quiescent solution at constant temperature, respectively. Fickian diffusion is defined as the following:

$$
\frac{\partial C_{i}}{\partial t}=D_{i}\left(\frac{\partial^{2} C_{i}}{\partial x^{2}}+\frac{\partial^{2} C_{i}}{\partial z^{2}}\right)
$$

where $C_{i}$ and $D_{i}$ represent the concentration and diffusion coefficient of a redox species, $i$, in solution. The direction of nanowire width is defined as ' $x$ ' while ' $z$ ' is the direction of electrode height. The diffusion regime achieved is assumed to be consistent along the length of the electrode. For the 2D model, the current density is converted by extrapolating the flux for the rectangle along the length of the electrode.

\subsubsection{D model}

A three-dimensional nanowire can be represented as a block of $50 \mathrm{~nm} \times 100 \mathrm{~nm} \times 45 \mu \mathrm{m}$. Due to symmetry, using one quarter of the block is sufficient. As with the 2D model, the domain is again set to be large enough so that the reaction of interest is at the centre of the domain and is unaffected by the bulk conditions at the domain edge. Fickian diffusion in 3D is defined as:

$$
\frac{\partial C_{i}}{\partial t}=D_{i}\left(\frac{\partial^{2} C_{i}}{\partial x^{2}}+\frac{\partial^{2} C_{i}}{\partial y^{2}}+\frac{\partial^{2} C_{i}}{\partial z^{2}}\right)
$$

Where ' $\mathrm{x}$ ' is again in the direction of nanowire width, ' $y$ ' is the direction of the nanowire length and ' $\mathrm{z}$ ' is the direction of nanowire height. 
The current is estimated from the models by use of the electroanalytical Butler-Volmer equation. [35] This expresses the current density as proportional to a heterogeneous rate constant $\mathrm{k}_{0}\left(\mathrm{~m} \cdot \mathrm{s}^{-1}\right)$ for the rate of electron transfer:

$$
i_{\text {loc }}=n F k_{0}\left(c_{\text {red }} \exp \left(\frac{\left(n-\alpha_{c}\right) F \eta}{R T}\right)-c_{o x} \exp \left(\frac{\left(-\alpha_{c}\right) F \eta}{R T}\right)\right)
$$

where $n$ is the number of electrons exchanged, $F$ is Faraday's constant, $k_{0}$ is the heterogeneous rate constant, $C_{r e d}$ and $C_{o x}$ are concentration $\left(\mathrm{mol} . \mathrm{m}^{-3}\right)$ of the reduced and oxidised species in the bulk and at the electrode surface with respect to time, respectively, $\alpha_{c}$ is the cathode exchange coefficient, $\eta$ is the overpotential, $R$ is the gas constant, and $T$ is temperature $(\mathrm{K})$.

The agreement between the extracted current from both models is expressed as the $2 \mathrm{D}$ current as a percentage of the $3 \mathrm{D}$ current

$$
\text { Agreement }=\frac{2 D \text { Current }}{3 D \text { Current }} \times 100
$$

The IDEs are modelled in generator-collector mode. An important parameter for evaluating the performance of an interdigitated electrode is the collection efficiency, which expresses the current at the collector electrodes as a percentage of the current at the generator electrode, given by the equation;

$$
\text { Collection Efficiency }=\frac{i_{c}}{i_{g}} \times 100
$$

where $i_{c}$ is the collector current and $i_{g}$ is the generator current.

As previously mentioned in the 2D model approach, the current density is converted by extrapolating the flux for the rectangle along the length of the electrode, while the 3D model extracts a current from the entire electrode surface area. Consequently mesh quality is of great importance to reduce any errors associated with the currents extracted from the model. A higher mesh density is required at the nanowire surface and region of high concentration gradient (Fig. S1). The mesh is refined until the current changes by $<1 \%$ between refinements. Simulations were resolved iteratively until a convergence error less than $1 \%$ error was achieved. 


\section{Results and Discussion}

\subsection{Device Characterisation}

Silicon chip sensor devices were fabricated as described in the experimental section. Fig. 1A shows an optical image of such a chip with six nanowire working electrodes (highlighted in red box) on each chip and also including counter and reference electrodes (blue box), contact pads (black boxes) and interconnection tracks. Fig. 1B and 1C show a SEM and AFM images of a single nanowire of $300 \mathrm{~nm}$ width. From the SEM analysis, the width of the nanowire was determined to be $325 \mathrm{~nm}$, approximately $10 \%$ larger than designed, and we attribute this increase to possible diffraction effects arising during e-beam patterning. The SEM characterisation further showed that the passivation layer had been completely removed from electrode surfaces and that good alignment and electrical connection existed between the electrodes and the overlaid interconnection tracks. AFM analysis of the nanowire exhibiting an average nanowire height of $65 \mathrm{~nm}, 5 \mathrm{~nm}$ more than the expected height of $60 \mathrm{~nm}$. This can be attributed to a slight over-etching of the underlying $\mathrm{SiO}_{2}$ layer undertaken to ensure that no SiN remained on the nanowire surface. These electrode dimensions were then included in the simulations that were used to compare with experimental results.
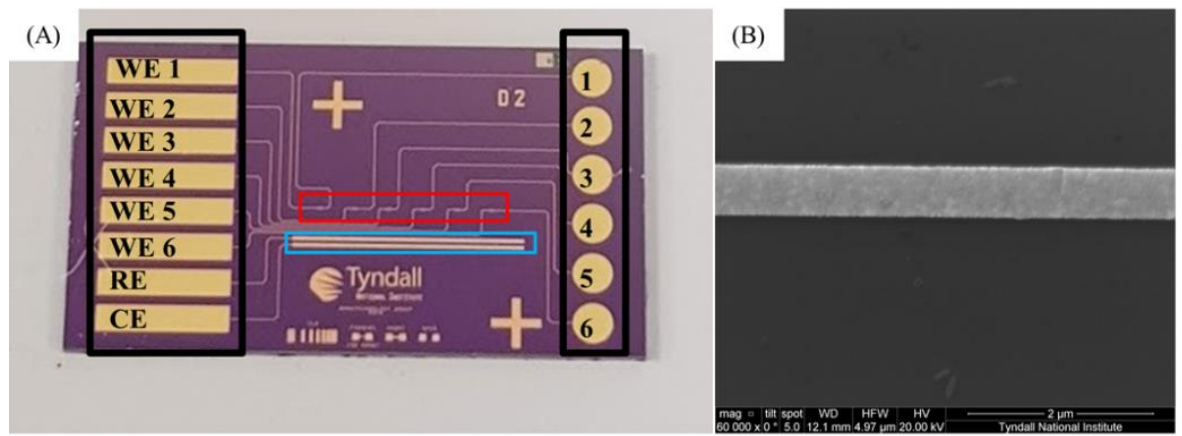

(C)
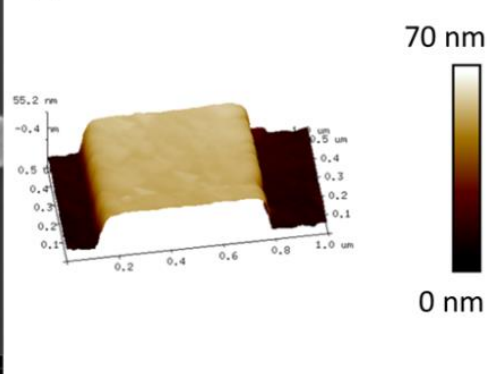

Fig. 1 (A) Image of silicon chip sensor device showing the working electrodes (red box), reference and counter electrodes (blue box) and contact pads for SD connector (LHS black box) and pins (RHS black box) (B) SEM of single nanowire of $325 \mathrm{~nm}$ width. (C) AFM of single nanowire of $65 \mathrm{~nm}$ height

\subsection{Single Nanowire}

Fig. 2A shows the simulated CV and extracted currents for $1.0 \mathrm{mM} \mathrm{FcCOOH}$ in $10 \mathrm{mM}$ PBS at a scan rate of $100 \mathrm{mV} / \mathrm{s}$. The $\mathrm{CVs}$ exhibit quasi steady-state behaviour, due to radial diffusion profiles at the nanowire dominating in the nano-regime, as expected. The current 
magnitude was observed to increase with increasing nanowire width; in line with an increased surface area. The slight hysteresis and diffusion type peaks were a result of the fitting methods in the 2D simulations. By contrast, Fig. 2B presents the same experimental conditions but simulated using a 3D approach. It can be observed that the hysteresis has decreased, and the shapes of the CVs have become more steady-state. In addition, the current magnitude for each nanowire width has increased compared to the 2D simulations. A significant difference in the agreement of extracted currents obtained using the $2 \mathrm{D}$ and $3 \mathrm{D}$ models (from equation 5) was observed; ranging from $89.2 \%$ to $87.5 \%$ agreement for 100 and $500 \mathrm{~nm}$ wide wires, respectively. To provide insight on this, experiments were undertaken at fabricated nanowire devices using the same experimental conditions as used in the simulations. Fig. $2 \mathrm{C}$ shows the experimental $\mathrm{CV}$ results obtained at single nanowire electrodes, of increasing width $(100-500 \mathrm{~nm})$ using a scan rate of $100 \mathrm{mV} / \mathrm{s}$. The CVs exhibited steady-state time independent behaviour in line with previous reports. [32] The slight hysteresis arose from the moderate scan rate used and was observed to decrease with decreasing scan rate. The current magnitude is again observed to increase with increased nanowire width, as expected. To compare simulated and experimental data, both simulated extracted and experimental peak maximum steady-state currents were plotted for each nanowire width; see Fig. 2D. A very good agreement between the experimental and 3D simulation data was observed with the slight discrepancies attributed to slight variations in the nanowire fabrication process. The similarity of the results confirm the accuracy our developed 3D model. Also it is observed that the experimental results diverge from the 2D simulated currents in a manner similar to the $3 \mathrm{D}$ simulations. 


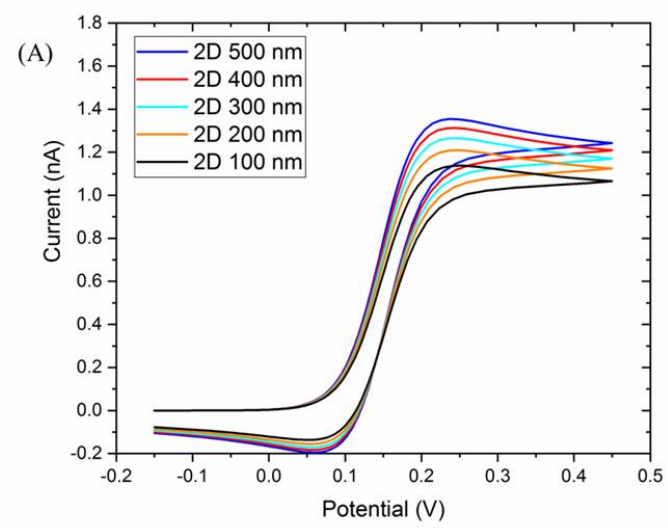

(B)
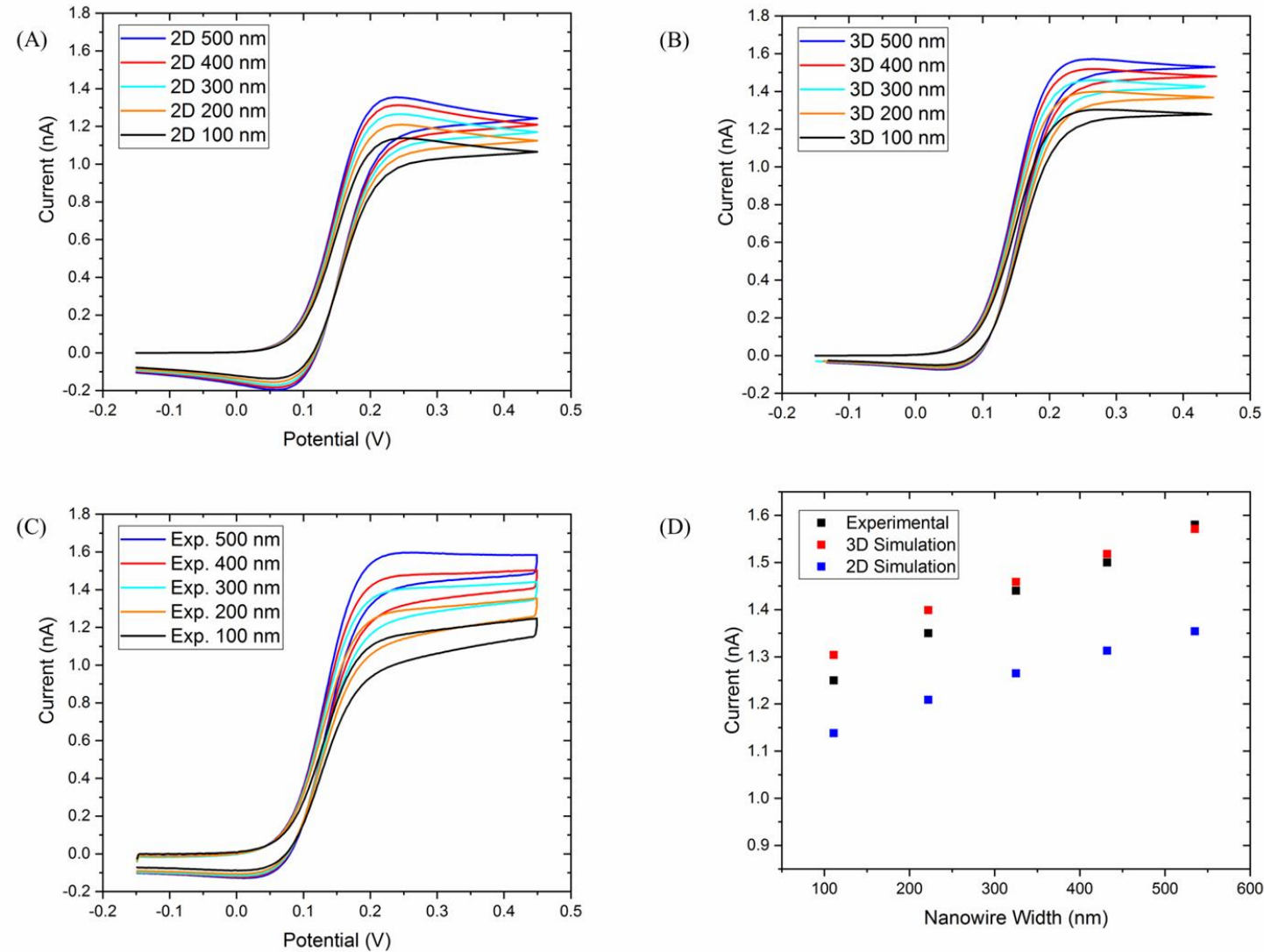

(D)

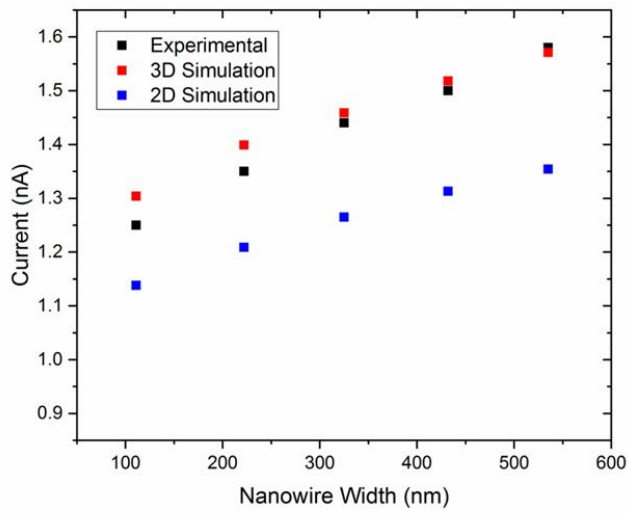

Fig. 2 Cyclic voltammograms of $1 \mathrm{mM} \mathrm{FcCOOH}$ at $100 \mathrm{mV} / \mathrm{s}$ for single nanowire of widths $100-500$ $\mathrm{nm}$ obtained (A) from 2D simulation; (B); from 3D simulation; (C) from experimental results. (D) Plot of peak currents versus electrode width for 2D simulation, 3D simulation and experimental data.

To understand this divergence, we looked at how the different simulations were undertaken. The 2D diffusion domain approach simulates mass transport to the electrode in 2 dimensions and extrapolates the current by integrating along the length of a nanowire. Therefore the current increases linearly with increasing electrode length. This is illustrated using the blue arrows confined within the blue box as highlighted in the SEM image presented in Fig. 3A. Fig. 3B shows a side elevation cross sectional 2D simulation plane for a single nanowire, exhibiting the molecular depletion zone at the nanowire and the radial molecular diffusion profiles; typically shown for a nanoscale electrode. [36] By contrast, Fig. 3C shows a plan view ( $\mathrm{x}, \mathrm{y}$ plane) of a 3D simulation undertaken for a single nanowire. As can be seen the depletion zone exists along the length of the nanowire in agreement with the $2 \mathrm{D}$ simulations but there is added depletion around each nanowire terminus which is not accounted for by the 2D model. These additional diffusion zones drive molecular diffusion to the nanowire 
termini and are illustrated using the red arrows in the SEM image; while the simulation domain is represented by the red and blue boxes combined as highlighted in Fig. 3A. Thus the 3D model includes diffusion to the electrode termini, and as such has a higher predicted current for a given electrode length, than the 2D. We hypothesise that it is this molecular diffusion, at the nanowire termini, that explains the discrepancy between the 2D and 3D simulations and also why the $3 \mathrm{D}$ simulations closely map onto the experimental data. If this hypothesis is correct, then the impact of "nanowire termini diffusion" effects on extracted current should diminish with increasing nanowire length as their proportional contribution to the overall measured currents would decrease.

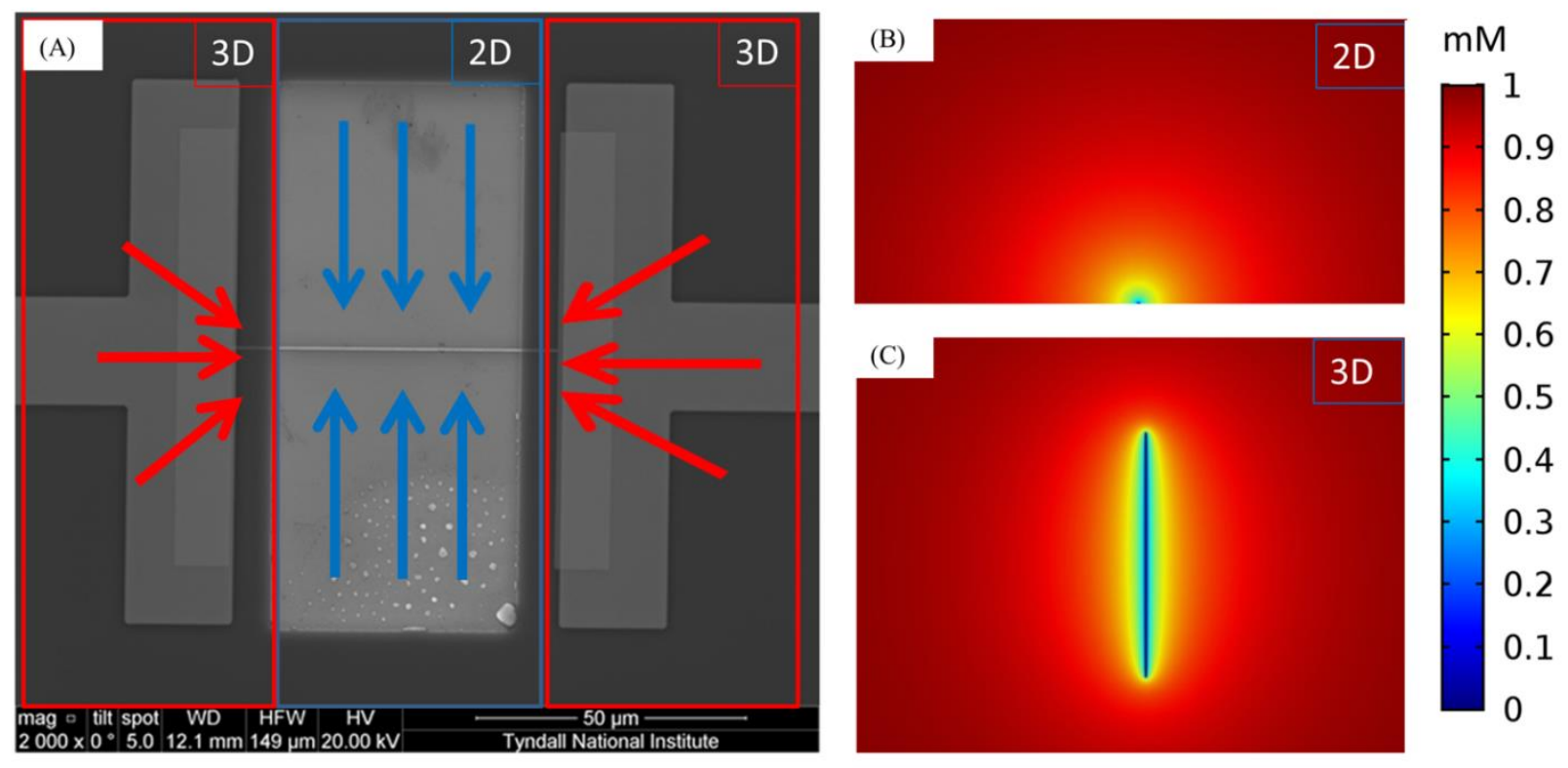

Fig. 3 (A) SEM image of nanowire and interconnection tracks. The area modelled by the 2D simulation is indicated by the blue box, while the red boxes highlight the additional areas modelled by the $3 \mathrm{D}$ simulation. (B) Cross sectional view of $2 \mathrm{D}$ simulation of $1 \mathrm{mM} \mathrm{FcCOOH}$ concentration profile at a single nanowire. (C) Plan view of 3D simulation of $1 \mathrm{mM} \mathrm{FcCOOH}$ concentration profile at a single nanowire

To probe this hypothesis, a theoretical study of the effects of nanowire electrode dimensions was undertaken by varying the length of the nanowire over the range of 15 to 1000 microns while retaining the width at $100 \mathrm{~nm}$ and height at $60 \mathrm{~nm}$, respectively. Fig. 4A shows the simulated CVs for a single nanowire electrode obtained using the 2D model for different electrode lengths. The peak current magnitude is seen to increase with increasing electrode length. This is inherent to the 2D approach, since the current is extrapolated directly by 
multiplying by the length of the electrode. Similarly Fig. 4B shows the simulated CVs obtained for a single nanowire electrode using the 3D model. Again, the current magnitude is seen to increase with increasing electrode length. The extracted peak current is again higher than the 2D model, due to diffusion effects at the electrode termini. Fig. 4C shows the relationship between the $2 \mathrm{D}$ and $3 \mathrm{D}$ extracted currents plotted as a percentage agreement versus electrode length. The 2D and 3D model are in greater agreement as electrode length increases, for example the 2D model predicted current has a $74 \%$ agreement with the $3 \mathrm{D}$ model at a nanowire length of $15 \mu \mathrm{m}$ while it has a $97 \%$ agreement with the 3D model at $1000 \mu \mathrm{m}$ length. As hypothesised, the current contribution attributed to the molecular diffusion occurring at nanowire termini to overall current decreased with increased electrode lengths, in agreement with the literature. [23] To further confirm that these differences arise from nanowire termini diffusion, a truncated 3D domain designed to mimic the region modelled by the 2D, by supressing termini diffusion (i.e., Fig 3A blue box) was undertaken and presented in Fig. S2. The extracted peak currents for these simulations were identical to those obtained using a 2D model approach. These results presented in Fig 4 show that the 2D approach is a suitable method for modelling band electrodes as they approach the macroscale, but the termini effects lead to significant difference from the 3D model when the ratio between length and width is less than 3 order of magnitudes (, i.e., L/W $<1000$.
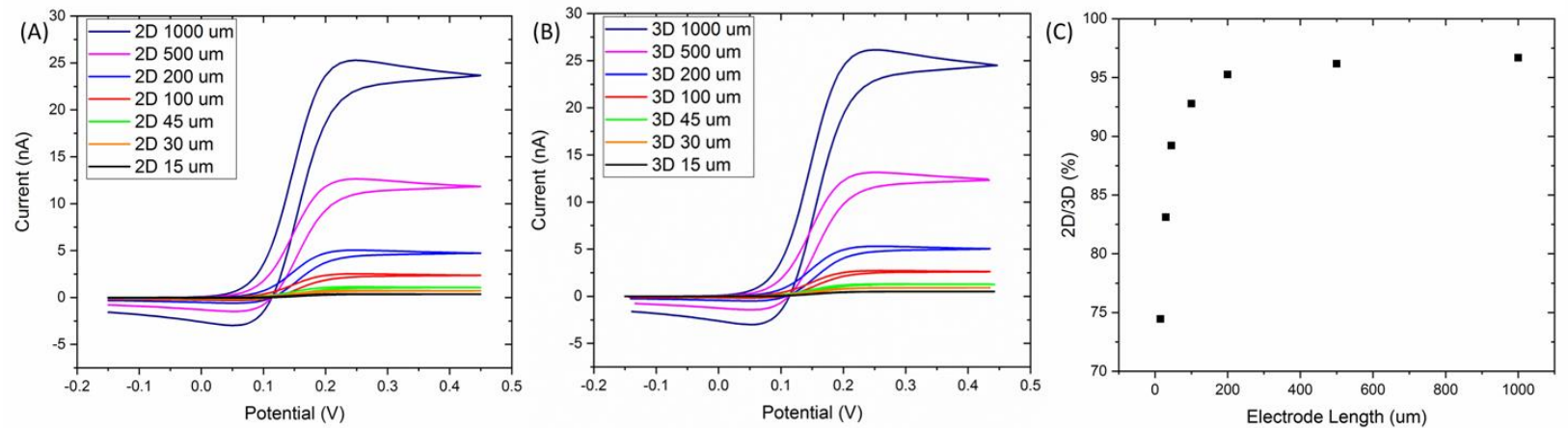

Fig. 4 Theoretical cyclic voltammograms of $1 \mathrm{mM} \mathrm{FcCOOH}$ at $100 \mathrm{mV} / \mathrm{s}$ for single nanowire of length 15 to 1000 micron for (A) 2D simulation and (B) 3D simulation; (C) Plot of 2D current over 3D current as a percentage vs electrode length.

\subsection{Nanowire Arrays}


Moving to arrays of nanowires, the limitations of the 2D diffusion domain approach were also explored with these configurations. Fig. 5A shows CVs simulated using both 2D and 3D models and electrochemically measured in $1 \mathrm{mM} \mathrm{FcCOOH}$ in $10 \mathrm{mM}$ PBS at $100 \mathrm{mV} / \mathrm{s}$ for an array of three nanowires (100 $\mathrm{nm}$ wide and $45 \mu \mathrm{m}$ length) separated by a gap of 15 microns. For the 2D model, an array of 3 nanowires yielded a current of $2.79 \mathrm{nA}$ rather than $3.39 \mathrm{nA}$ ( $3 \mathrm{x}$ that of a single nanowire, $1.13 \mathrm{nA}$ Fig $2 \mathrm{C}$ ), indicating that some diffusional overlap of the individual nanowire diffusion profiles is occurring and planar begins to dominate. This trend in diffusional overlap is also seen in both the 3D simulations and experimental data. As for the single nanowire data presented in Fig 2, the extracted 3D model data most closely maps onto the experimental data. The 2D model yielded a lower current than that of the 3D model, with a peak current $\sim 83 \%$ the magnitude of the 3D current. This is a greater difference than was observed for the 2D/3D (agreement $\sim 89 \%$ ) for a single nanowire Fig 2D. The increase in disparity can again be attributed to molecular diffusion occurring at the nanowire termini. Fig. 5B shows a typical concentration profile of FcCOOH in a plan view (x,y plane) of the electrodes from the 3D model (a corresponding profile from the 2D model is provided in Fig. S3). There is significant diffusional overlap between the 3 nanowires and the overall profile of the array is behaving as a larger microelectrode of 30.3 micron (the distance separating the outer electrodes).
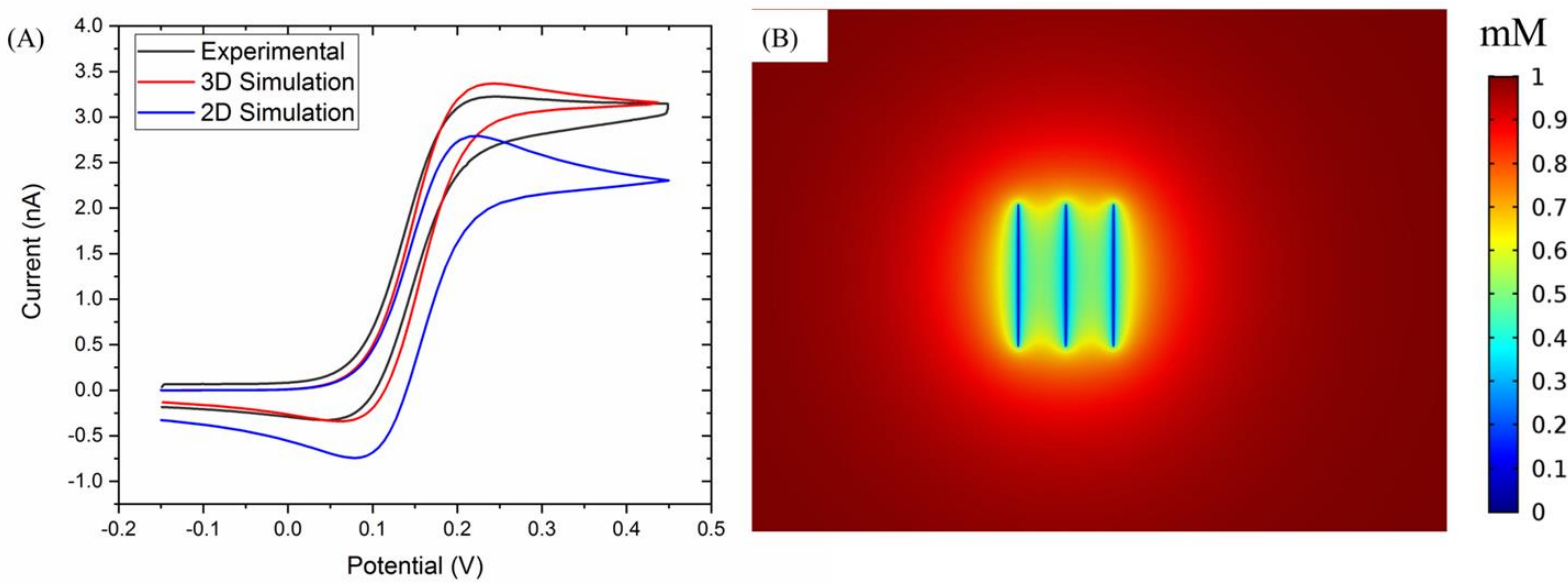

Fig. 5 (A) Cyclic voltammograms of $1 \mathrm{mM} \mathrm{FcCOOH}$ at $100 \mathrm{mV} / \mathrm{s}$ for array of $3 \times 100 \mathrm{~nm}$ wide nanowires separated by 15 micron obtained from 2D simulation, 3D simulation and experimental data (B) Plan view of 3D simulation of $\mathrm{FcCOOH}$ concentration profile at array of 3 nanowires 
From the data presented in Fig 5, it may be assumed that with increasing numbers of nanowires in an array, the greater the 2D simulations will diverge from both 3D simulations and experimental results. This assumption would have significant implications for nanoelectrode design architecture. To confirm this assumption the models were extended to enable a theoretical study of changing the number of electrodes in a nanowire array to be undertaken. Fig. 6A shows the simulated CVs for increasing numbers of nanowires in an array using the 2D model, while the 3D model data is presented in Fig. 6B. The number of electrodes in the array (n) is varied from 1 to 10, with a gap of $15 \mu \mathrm{m}$ maintained between each electrode; while the lengths and widths were kept constant at 45 microns and $100 \mathrm{~nm}$, respectively. With additional electrodes, the current magnitude is seen to increase, using both simulation methods, as would be expected. The CVs become more diffusion limited with increasing numbers of electrodes exhibit behaviour similar to a larger single microelectrode. The current magnitudes extracted from the 3D model are greater than that of the $2 \mathrm{D}$ for each value of $\mathrm{n}$ as seen in Fig. 6C; where all the extracted peak current are plotted for comparison.

Fig. 6D shows the relationship between the 2D and 3D extracted currents plotted as a percentage agreement versus $\mathrm{n}$. The percentage agreement between the two models decreases with each additional nanowire from $89.2 \%$ for 1 nanowire down to $81 \%$ for 10 nanowires. However, the degree of divergence between the 2D and 3D models decreases with each additional nanowire, with an $3.4 \%$ difference moving from 1 to 2 nanowire, but only a $0.6 \%$ difference moving from 5 electrodes to 10 nanowires. Since the array behaves as a microelectrode with a width the size of the array footprint, each additional electrode adds a fixed additional width to the pseudo microelectrode. This fixed width increase is a smaller percentage of the overall array footprint, per additional nanowire, and as the divergence between the 2D and 3D model is linked to the electrode width (Fig. 2C); this leads to a reduced increase in divergence between the 2D and 3D models. 

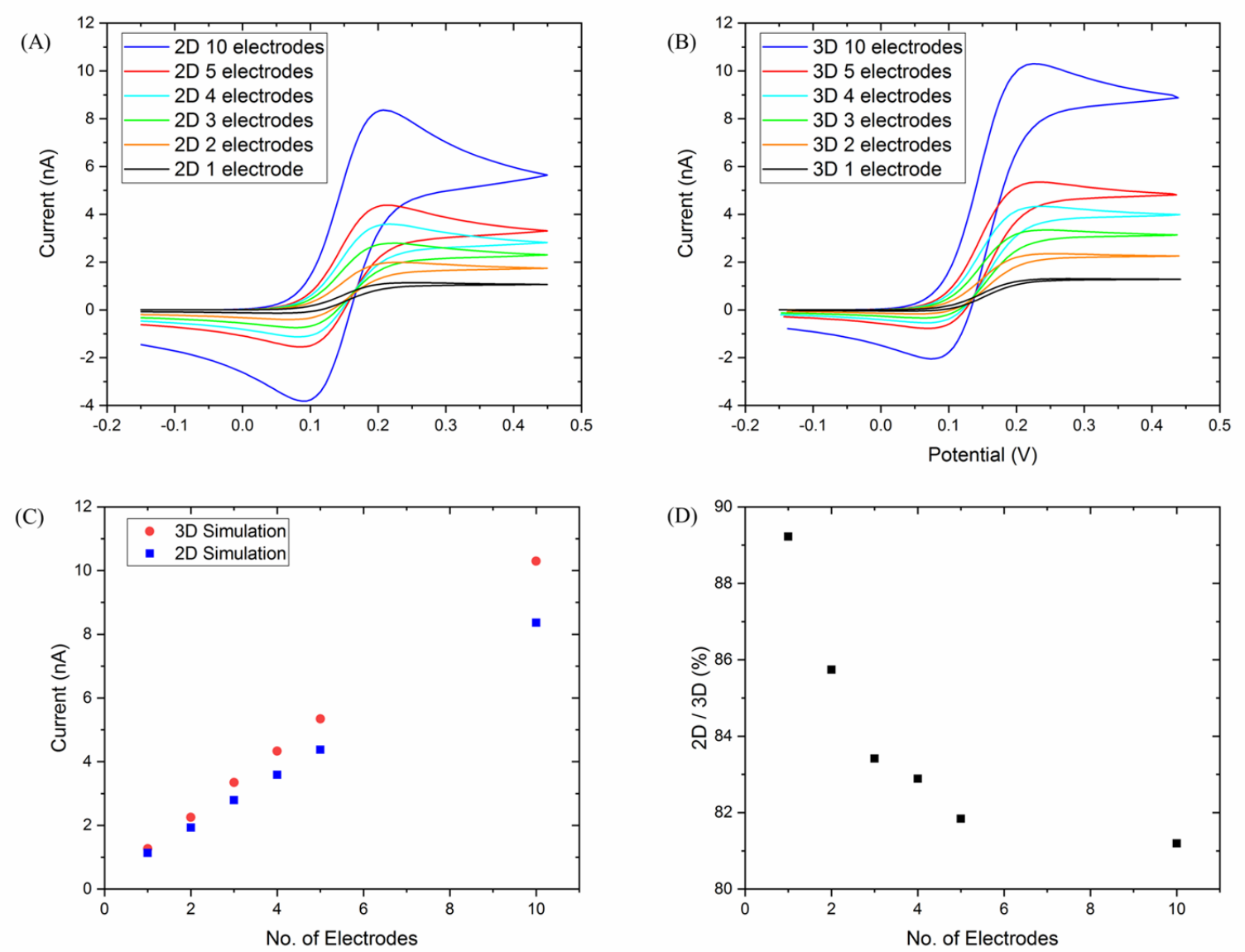

Fig. 6 Cyclic voltammograms of $1 \mathrm{mM} \mathrm{FcCOOH}$ at $100 \mathrm{mV} / \mathrm{s}$ for array of nanowires separated by 15 micron for 1 to 10 electrodes in the array from (A) 2D simulation and (B) 3D simulation; (C) Plot of current vs number of electrodes in the array for 2D and 3D currents; (D) Plot of 2D current over 3D current as a percentage vs number of electrodes in the array.

The effect of altering the separation (or gap) between nanowires in an array was explored using $1.0 \mathrm{mM} \mathrm{FcCOOH}$ in $10 \mathrm{mM}$ PBS at $100 \mathrm{mV} / \mathrm{s}$ and are presented for the 3D model in Fig. 7 (and in the supporting information Fig. S4 for the 2D model). A large range of nanowire separations from $100 \mathrm{~nm}$ up to $200 \mu \mathrm{m}$ were simulated. Fig. 7A shows the simulation results of an array of three nanowires each separated by a gap of $100 \mathrm{~nm}$. As shown by the solid blue colour (representing $0 \mathrm{mM}$ of $\mathrm{FcCOOH}$ ), there is complete overlap of the individual nanowire diffusion profiles occurring over the entire array and thus the array consequently behaves as a larger, $500 \mathrm{~nm}$ wide electrode. With increasing separations up to $5 \mu \mathrm{m}$ it was observed that there was still a high level of diffusion overlap between the nanowires for the experimental conditions being simulated, see Fig. 7B. When the separation 
was increased above 5 microns, the degree of diffusional overlap between individual nanowires begins to decrease until the nanowires within an array become fully diffusionally independent. Fig. 7C shows a situation where the individual nanowires are separated by 200 $\mu \mathrm{m}$ and are clearly diffusionally independent. Fig. 7D shows the percentage agreement, with increasing electrode separations, for the extracted current magnitudes obtained using both the 2D and 3D models. There are two trends in the data - the first is at low separations between the nanowires, i.e., ranging from $100 \mathrm{~nm}$ to $5 \mu \mathrm{m}$. In this range, the percentage agreement between the current magnitudes of the 2D and 3D models decrease with increasing separation. At very small inter-electrode separation, there is a higher, almost total level of diffusional overlap. For example, the diffusion concentration profiles occurring at a $3 \times 100$ $\mathrm{nm}$ wide nanowires separated by $100 \mathrm{~nm}$, shown in Fig. 7A, is comparable to that of a 500 $\mathrm{nm}$ wide single nanowire. By contrast, with increasing electrode separation, 5 microns and above, a second trend emerges as the degree of overlap begins to decrease. The concentration profile plot in Fig. 7B shows an array with 5 micron separation. The concentration profile shows differing degrees of diffusional overlap occurring along the length of the electrode. The concentration regimes around the individual electrode termini are visible indicating a lower diffusional overlap, while a higher degree of overlap remaining along the length of the electrode. Since the $2 \mathrm{D}$ model extrapolates a fixed value for the entire length of the electrode, this leads to the larger divergence in agreement between the $2 \mathrm{D}$ and 3D models. By increasing the separation between nanowires further, the corresponding diffusional overlap between the nanowires along the entire length of the nanowire begins to decrease significantly and the array begins to behave more and more like independent electrodes and thus the agreement between the extracted currents begins to increase again. At large inter-electrode gaps, the electrodes ultimately become diffusionally independent as shown in Fig. 7C. For the diffusionally independent arrays, the arrays behave as three single nanowires, which is reflected by the agreement between the $2 \mathrm{D}$ and $3 \mathrm{D}$ models, returning to $89 \%$, the same as a single nanowire as presented in Fig. 4, above. 

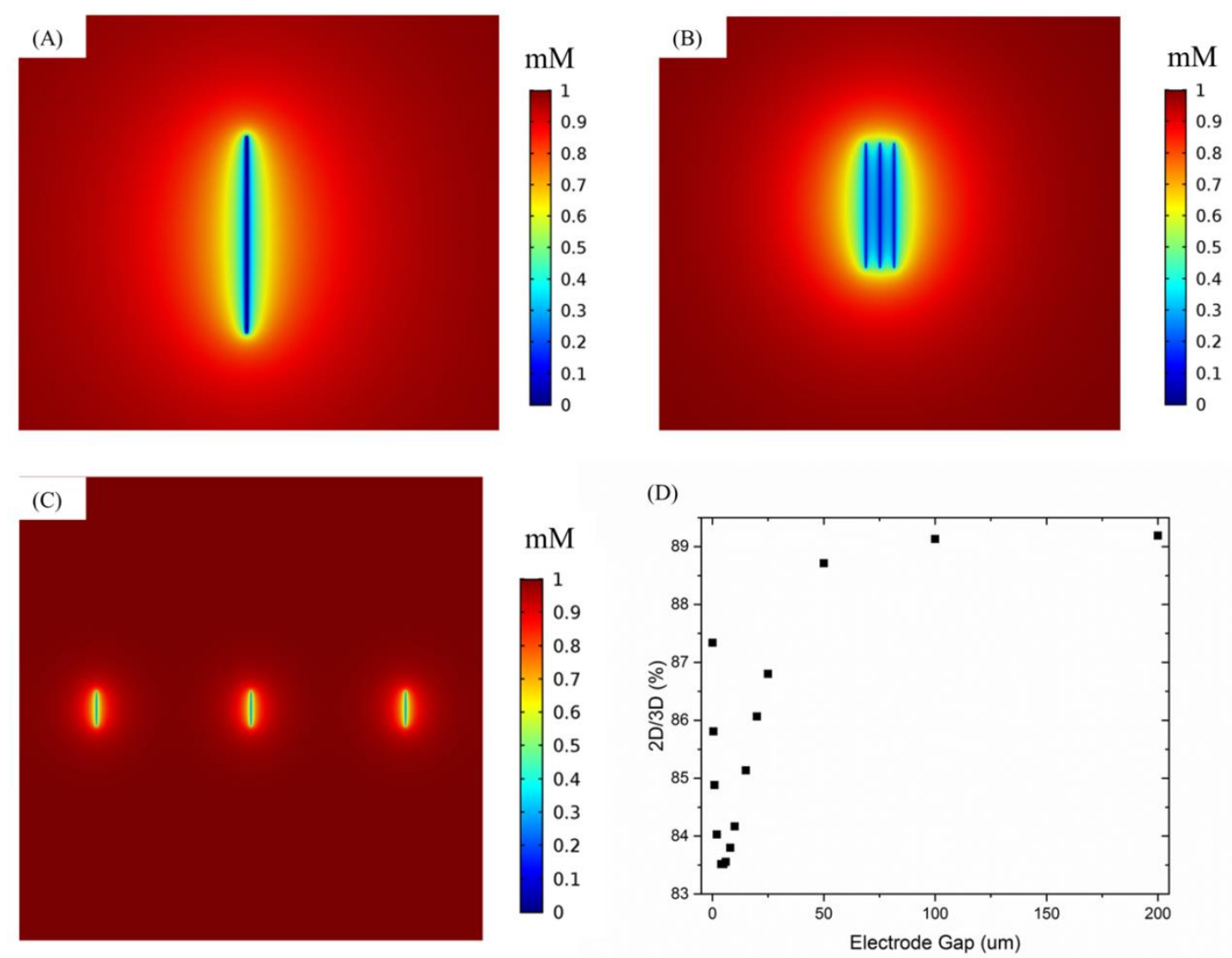

Fig. 7 (A) Plan view of 3D simulation of $1 \mathrm{mM}$ FcCOOH concentration profile at array of 3 nanowires with an inter electrode gap of (A) $100 \mathrm{~nm}$ (B) $5 \mu \mathrm{m}$ and (C) $200 \mu \mathrm{m}$; (D) Plot of 2D current as a percentage of $3 \mathrm{D}$ current versus inter electrode seperation

\subsection{Interdigitated Nanowire Arrays}

The simulation and experimental approach was also applied to solid state generator-collector electrochemistry. In this work, we fabricated two interdigitated electrodes where the generator comprises of single nanowire electrode placed interstitially between two (electrically connected) collector electrodes; see inset Fig. 8A, denoted as an electrode triplet. In this manner, electrochemical species generated at the generator electrode will diffuse outwards to, and be collected by, the two collector electrodes. By judicious selection of applied voltages to the generator and collector electrodes, the measured current signals could be significantly increased by means of redox cycling. Simulations (both 2D \& 3D) and 
experiments were undertaken for different inter-electrode gaps varying from 200 to $1000 \mathrm{~nm}$. The inverse set-up (2 generators and 1 collector) is presented in Fig. S5.

Fig. 8A shows the CVs and extracted currents for $1.0 \mathrm{mM} \mathrm{FcCOOH}$ in $10 \mathrm{mM}$ PBS at 100 $\mathrm{mV} / \mathrm{s}$ for an electrode triplet operating in generator-collector mode using the $2 \mathrm{D}$ model. The inner generator electrode was cycled from $-0.15 \mathrm{~V}$ to $0.45 \mathrm{~V}$ while the outer collector electrodes were held at a constant $-0.15 \mathrm{~V}$. The $\mathrm{CVs}$ for both the generator and collector are presented for a range of inter-comb gaps. It was observed that the magnitude of the generator current was greater than that of a single nanowire electrode regardless of the interdigitated gap. This was due to redox cycling occurring where $\mathrm{FcCOOH}$ oxidised to $\mathrm{FcCOOH}^{+}$at the generator diffused to and was reduced back to $\mathrm{FcCOOH}$ at the collector, where on it can once again diffuse to the generator and be re-oxidised. The inset shows an increased concentrations of $\mathrm{FcCOOH}$ at the collectors compared to the bulk solution. The current magnitudes of both generator and collector electrodes increased with decreasing electrode gap as the collection efficiency of the electrode system increased. As the gap between the combs increased, a larger proportion of the $\mathrm{FcCOOH}^{+}$was free to diffuse out into the bulk solution instead of to the collector electrodes (for reduction). A similar trend was predicted by the 3D model, shown in Fig. 8B. The current magnitude of both generator and collector electrodes also increased with decreasing electrode gap. While the 3D model yielded a slightly higher generator current, there is much closer agreement with regard to the $2 \mathrm{D}$ model when compared to a non-GC single nanowire devices. The current at the collector electrode was similar in magnitude but slightly lower for the 3D model when compared to the 2D model. The inset in the figure shows a schematic of the redox cycling approach. Fig. 8C shows the experimental data obtained from fabricated triplet electrode devices, where the measured current magnitudes are observed to be in good agreement with the predicted values from both simulation approaches. Fig. 8D presents a plot of collection efficiency of the current obtained using the 2D, 3D and experimental data; determined using equation 6.

As expected, and in line with the current magnitudes, the collection efficiency decreased with increasing electrode separation gap in all cases. Again, the experimental data was observed to map most closely onto the 3D simulation results. By contrast to the single and nanowire array electrodes operating in non-GC mode, presented heretofore, the 2D simulations have higher predicted collection efficiencies for all electrode gaps when compared to the $3 \mathrm{D}$ and experimental results. This over-estimation of collection efficiency can again be attributed to molecular diffusion. When operating in GC mode, it is now the case that the 2D model does 
not allow molecules to diffuse away from (rather than to as in the case of CV presented above) the nanowire termini in the y-axis and assumes all the molecules undergo Redox cycling. The 3D model takes into account this diffusion away from the electrode termini and thus more closely estimates an extracted current in line with the experimental data. Consequently it is apparent that molecular diffusion at nanowire termini also impacts generator collector electrochemical measurements. If this hypothesis is correct, as with nanowires in non-GC mode, the impact of "nanowire termini diffusion" effects on extracted current should again diminish with increasing nanowire length as their proportional contribution to the overall measured currents would decrease.
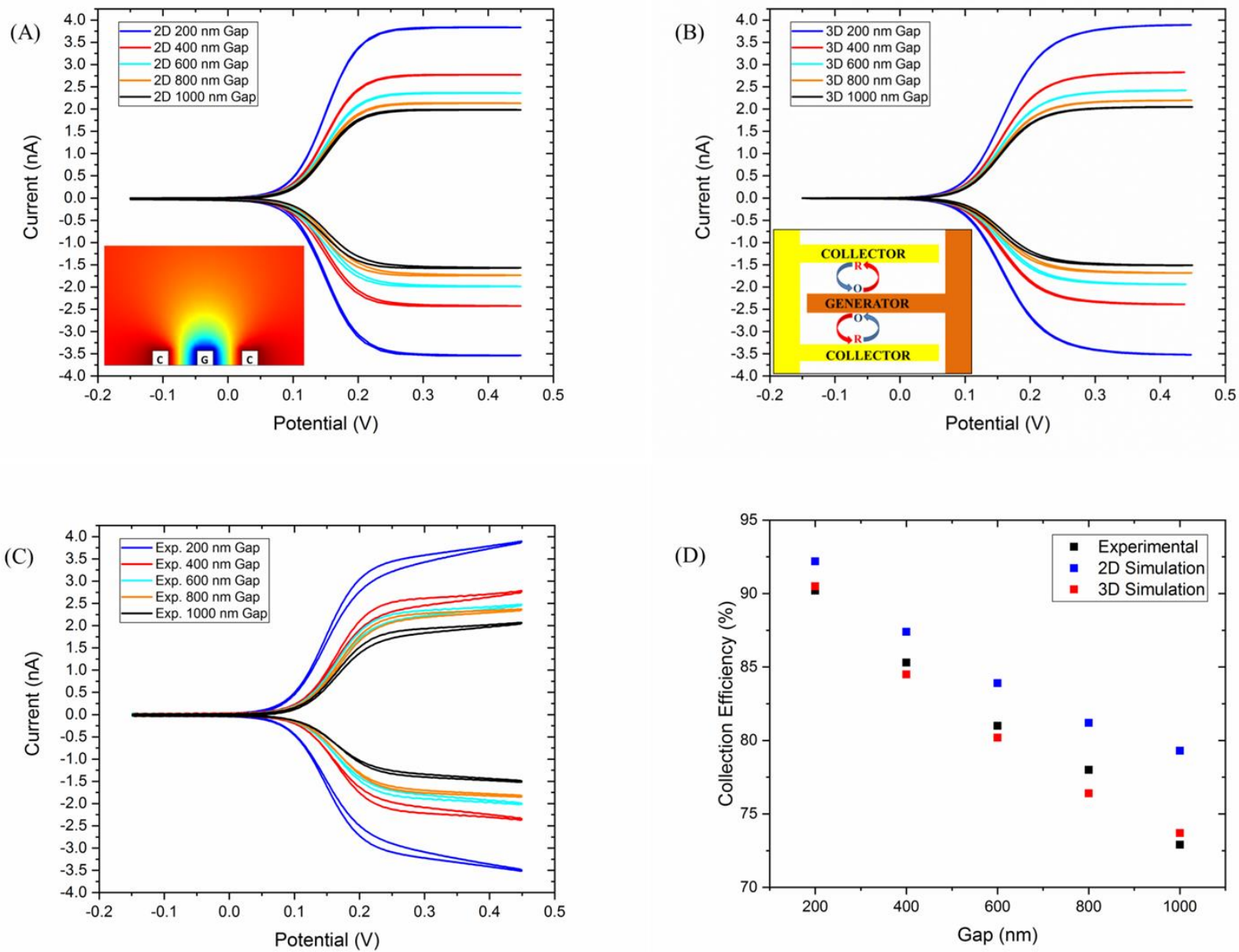

Fig. 8 Cyclic voltammograms of $1 \mathrm{mM} \mathrm{FcCOOH}$ at interdigitated triplet electrode comprising of a single generator nanowire and two external collector nanowires in GC mode at a range of interelectrode gaps for (A) 2D simulations (inset: side-on concentration profile of $\mathrm{FcCOOH}$ at generator(G) and collector (C) electrodes), (B) 3D simulations (inset: schematic showing redox cycling between generator and collectors), and (C) experimental data. The potential at the generator 
was cycled from -0.15 to $0.45 \mathrm{~V}$ while the collectors were held at $-0.15 \mathrm{~V}$. (D) Plot of collection efficiency versus inter electrode gap from 2D simulation, 3D simulation and experimental current.

To probe this hypothesis, a theoretical study of the effect of nanowire electrode length ranging from 15 to 200 microns (while retaining the nanowire width at $100 \mathrm{~nm}$ ) was undertaken. Fig. 9A shows the extracted simulated currents from the 2D model of a single generator and two collectors (described above) separated by $500 \mathrm{~nm}$ with a range of electrode lengths. For the 2D simulations, the current magnitudes for the generator and collector electrodes increased with increasing electrode length. As previously discussed, this is inherent to the 2D model since the currents are extrapolated via multiplication by the electrode length. In the 3D model, Fig. 9B, the current magnitudes were again observed to increase with electrode length, reflecting the increase in electrode surface area. For each given length, the generator current magnitudes were greater in the 3D model, arising from additional diffusion from the bulk to the nanowire termini. By contrast, the extracted current magnitude of the collectors were lower for the 3D model for all nanowire lengths, due to the molecular diffusion away from the collector termini into the bulk. The agreement between the current magnitudes obtained from the two models increased with increasing nanowire length increasing from $97.4 \%$ at $15 \mu \mathrm{m}$ length to $99.4 \%$ at $200 \mu \mathrm{m}$ length. As shown in Fig. 8D, the key discrepancy between the 2D and 3D model was in collection efficiency, with 2D overestimating this value compared with both the 3D model and experimental data. Fig. 9C shows the agreement in collection efficiencies predicted by both the 2D and 3D models versus electrode length. The collection efficiency of 2D is unchanged by electrode length, while the 3D model shows the collection efficiency increase with increasing electrode length tending towards that of the 2D. This is due to the diminishing effects of molecular diffusion at nanowire termini and the agreement between the models increases as discussed above. One can also conclude that at increasingly larger length scales, the 2D model approach, with reduced computer requirements, is adequate to provide an estimate of collector efficiency while 3D models will be required at shorted length scales. 

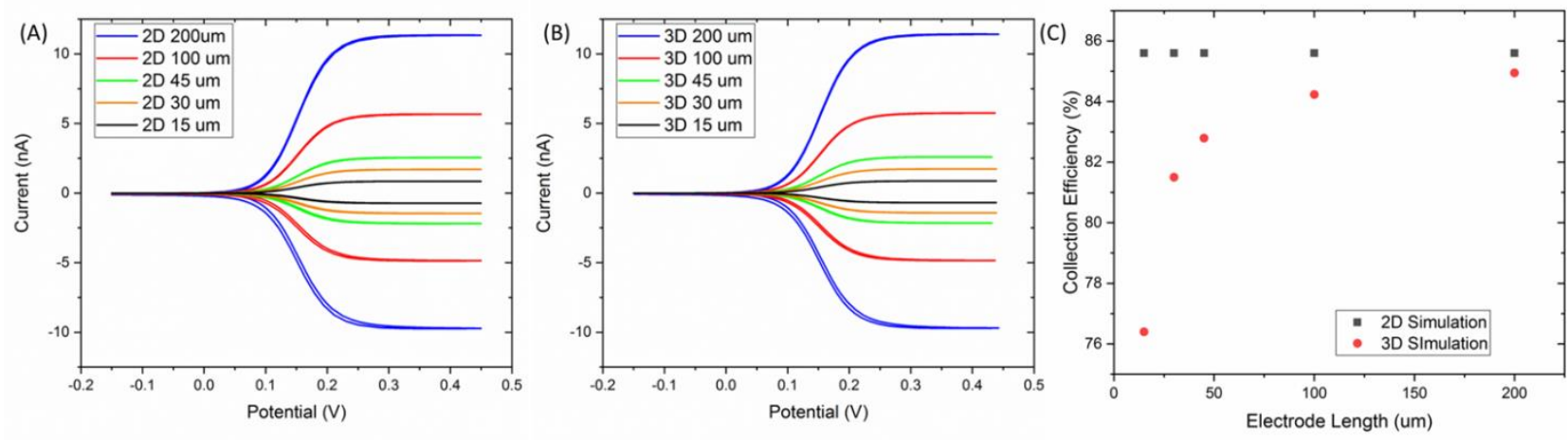

Fig. 9 Cyclic voltammograms of $1 \mathrm{mM} \mathrm{FcCOOH}$ at interdigitated array of a nanowire electrode tiplet GC mode with an inter-electrode gap of $500 \mathrm{~nm}$ for (A) 2D simulations and (B) 3D simulations for a range of electrode lengths from 15 micron to 200 micron (C)The potential at the generator was cycled while the collectors were held at $-0.15 \mathrm{~V}$. (D) Plot of collection efficiency versus inter electrode gap from 2D simulation, 3D simulation and experiemental current.

\section{Conclusion}

The 2D diffusion domain approach for modelling electroanalysis offers a valuable means of converting complex $3 \mathrm{D}$ calculations into more simple 2D estimates. The 2D approach offers a good prediction of current from the electroanalytical Butler-Volmer for protruding nanoband electrodes that have a length approaching the macroscale, requires reduced computational power and provide results quite rapidly. However, the 2D approach suffers from one serious limitation in that it ignores molecular diffusion to and from electrodes from the third dimension which affects extracted currents and electrochemical profiles from electrode geometries that are in the nano and ultra-microelectrode regime. This limitation is enhanced for arrays of nanowires with more termini for this diffusion to occur. In generator collector mode, the 2D model predicts higher collection efficiency due to a lack of diffusion in the y axis. We show that 3D simulation approach will provide a more accurate description of such device designs at all length scales and is significantly more accurate for single nanoelectrodes, arrays of nanoelectrodes and nanoelectrode based generator-collector electrochemistry up to $200 \mathrm{~nm}$ in length.

\section{Acknowledgements}


The authors would like to acknowledge funding from the Irish Research Council (Project GOIPG/2015/2925). This work has been supported in part by a research grant for the VistaMilk Centre Science Foundation Ireland (SFI); Department of Agriculture, Food and the Marine (DAFM) under Grant Number 16/RC/3835

\section{References}

[1] L.A. Wasiewska, I. Seymour, B. Patella, R. Inguanta, C.M. Burgess, G. Duffy, A. O'Riordan, Reagent free electrochemical-based detection of silver ions at interdigitated microelectrodes using in-situ $\mathrm{pH}$ control, Sensors and Actuators B: Chemical, 333 (2021) 129531.

[2] B. Patella, R.R. Russo, A. O'Riordan, G. Aiello, C. Sunseri, R. Inguanta, Copper nanowire array as highly selective electrochemical sensor of nitrate ions in water, Talanta, 221 (2021) 121643.

[3] B.N. Viada, L.M. Yudi, D.W.M. Arrigan, Detection of perfluorooctane sulfonate by ion-transfer stripping voltammetry at an array of microinterfaces between two immiscible electrolyte solutions, Analyst, 145 (2020) 5776-5786.

[4] B. Patella, M. Buscetta, S. Di Vincenzo, M. Ferraro, G. Aiello, C. Sunseri, E. Pace, R. Inguanta, C. Cipollina, Electrochemical sensor based on $\mathrm{rGO} /$ Au nanoparticles for monitoring $\mathrm{H} 2 \mathrm{O} 2$ released by human macrophages, Sensors and Actuators B: Chemical, 327 (2021) 128901.

[5] R. Daly, T. Narayan, H. Shao, A. O'Riordan, P. Lovera, Platinum-Based Interdigitated MicroElectrode Arrays for Reagent-Free Detection of Copper, Sensors, 21 (2021).

[6] I. Seymour, B. O'Sullivan, P. Lovera, J.F. Rohan, A. O'Riordan, Electrochemical detection of freechlorine in Water samples facilitated by in-situ pH control using interdigitated microelectrodes, Sensors and Actuators B: Chemical, 325 (2020) 128774.

[7] I. Seymour, B. O'Sullivan, P. Lovera, J.F. Rohan, A. O'Riordan, Elimination of Oxygen Interference in the Electrochemical Detection of Monochloramine, Using In Situ pH Control at Interdigitated Electrodes, ACS Sensors, 6 (2021) 1030-1038.

[8] C. Ma, N.M. Contento, L.R. Gibson, P.W. Bohn, Redox Cycling in Nanoscale-Recessed Ring-Disk Electrode Arrays for Enhanced Electrochemical Sensitivity, ACS Nano, 7 (2013) 5483-5490.

[9] S.-R. Kwon, K. Fu, D. Han, P.W. Bohn, Redox Cycling in Individually Encapsulated Attoliter-Volume Nanopores, ACS Nano, 12 (2018) 12923-12931.

[10] A. Wahl, K. Dawson, J. MacHale, S. Barry, A.J. Quinn, A. O'Riordan, Gold nanowire electrodes in array: simulation study and experiments, Faraday Discussions, 164 (2013) 377-390.

[11] N. Godino, X. Borrisé, F.X. Muñoz, F.J. del Campo, R.G. Compton, Mass Transport to Nanoelectrode Arrays and Limitations of the Diffusion Domain Approach: Theory and Experiment, The Journal of Physical Chemistry C, 113 (2009) 11119-11125.

[12] R.G. Compton, G.G. Wildgoose, N.V. Rees, I. Streeter, R. Baron, Design, fabrication, characterisation and application of nanoelectrode arrays, Chemical Physics Letters, 459 (2008) 1-17. [13] L.N. Nekrasov, Detection and identification of intermediate and final products of electrochemical reactions by means of the rotating ring-disc electrode method, Faraday Discussions of the Chemical Society, 56 (1973) 308-316.

[14] D. Han, L.P. Zaino, K. Fu, P.W. Bohn, Redox Cycling in Nanopore-Confined Recessed Dual-Ring Electrode Arrays, The Journal of Physical Chemistry C, 120 (2016) 20634-20641.

[15] E.O. Barnes, G.E.M. Lewis, S.E.C. Dale, F. Marken, R.G. Compton, Dual band electrodes in generator-collector mode: Simultaneous measurement of two species, Journal of Electroanalytical Chemistry, 703 (2013) 38-44. 
[16] C. Amatore, J.M. Savéant, D. Tessier, Charge transfer at partially blocked surfaces: A model for the case of microscopic active and inactive sites, Journal of Electroanalytical Chemistry and Interfacial Electrochemistry, 147 (1983) 39-51.

[17] I.J. Cutress, E.J.F. Dickinson, R.G. Compton, Analysis of commercial general engineering finite element software in electrochemical simulations, Journal of Electroanalytical Chemistry, 638 (2010) 76-83.

[18] J. Guo, E. Lindner, Cyclic Voltammograms at Coplanar and Shallow Recessed Microdisk Electrode Arrays: Guidelines for Design and Experiment, Analytical Chemistry, 81 (2009) 130-138.

[19] D. Li, C. Lin, C. Batchelor-McAuley, L. Chen, R.G. Compton, Electrochemical measurement of the size of microband electrodes: A theoretical study, Journal of Electroanalytical Chemistry, 840 (2019) 279-284.

[20] J. Xiong, Q. Chen, M.A. Edwards, H.S. White, Ion Transport within High Electric Fields in Nanogap Electrochemical Cells, ACS Nano, 9 (2015) 8520-8529.

[21] C. Ma, W. Xu, W.R.A. Wichert, P.W. Bohn, Ion Accumulation and Migration Effects on Redox Cycling in Nanopore Electrode Arrays at Low lonic Strength, ACS Nano, 10 (2016) 3658-3664.

[22] E.O. Barnes, G.E.M. Lewis, S.E.C. Dale, F. Marken, R.G. Compton, Generator-collector double electrode systems: A review, Analyst, 137 (2012) 1068-1081.

[23] J. Strutwolf, Computational Study of Chronoamperometry at Rectangular Microelectrodes, Electroanalysis, 17 (2005) 1547-1554.

[24] I.J. Cutress, R.G. Compton, Theory of square, rectangular, and microband electrodes through explicit GPU simulation, Journal of Electroanalytical Chemistry, 645 (2010) 159-166.

[25] E.J.F. Dickinson, H. Ekström, E. Fontes, COMSOL Multiphysics ${ }^{\circledR}$ : Finite element software for electrochemical analysis. A mini-review, Electrochemistry Communications, 40 (2014) 71-74.

[26] H.L. Woodvine, J.G. Terry, A.J. Walton, A.R. Mount, The development and characterisation of square microfabricated electrode systems, Analyst, 135 (2010) 1058-1065.

[27] D.E. Molina, A.S. Medina, H. Beyenal, C.F. Ivory, Design and Finite Element Model of a Microfluidic Platform with Removable Electrodes for Electrochemical Analysis, Journal of The Electrochemical Society, 166 (2019) B125-B132.

[28] M. Atighilorestani, A.G. Brolo, Comparing the Electrochemical Response of Nanostructured Electrode Arrays, Analytical Chemistry, 89 (2017) 6129-6135.

[29] D. Britz, J. Strutwolf, O. Østerby, Revisiting rectangular electrodes; a simulation study, Electrochimica Acta, 338 (2020) 135728.

[30] C. Batchelor-McAuley, R.G. Compton, Diffusion to a cube: A 3D implicit finite difference method, Journal of Electroanalytical Chemistry, 877 (2020) 114607.

[31] K. Dawson, A. Wahl, S. Barry, C. Barrett, N. Sassiat, A.J. Quinn, A. O'Riordan, Fully integrated onchip nano-electrochemical devices for electroanalytical applications, Electrochimica Acta, 115 (2014) 239-246.

[32] A.J.C. Wahl, I.P. Seymour, M. Moore, P. Lovera, A. O'Riordan, J.F. Rohan, Diffusion profile simulations and enhanced iron sensing in generator-collector mode at interdigitated nanowire electrode arrays, Electrochimica Acta, 277 (2018) 235-243.

[33] A. Piper, Ben M. Alston, D.J. Adams, A.R. Mount, Functionalised microscale nanoband edge electrode (MNEE) arrays: the systematic quantitative study of hydrogels grown on nanoelectrode biosensor arrays for enhanced sensing in biological media, Faraday Discussions, 210 (2018) 201-217. [34] D. Li, C. Batchelor-McAuley, L. Chen, R.G. Compton, Band Electrodes in Sensing Applications: Response Characteristics and Band Fabrication Methods, ACS Sensors, 4 (2019) 2250-2266.

[35] E.J.F. Dickinson, A.J. Wain, The Butler-Volmer equation in electrochemical theory: Origins, value, and practical application, Journal of Electroanalytical Chemistry, 872 (2020) 114145.

[36] D.W.M. Arrigan, Nanoelectrodes, nanoelectrode arrays and their applications, Analyst, 129 (2004) 1157-1165. 


\section{Supporting Information for}

\section{Comparison of 2D versus 3D diffusion analysis at Nanowire Electrodes: Finite element analysis and experimental study}

Benjamin O’Sullivan ${ }^{\mathrm{a}}$, Shane O’Sullivan ${ }^{\mathrm{a}}$, Tarun Naruyan ${ }^{\mathrm{a}}$, Han Shao ${ }^{\mathrm{a}}$, Bernardo Patella ${ }^{\mathrm{ab}}$, Ian Seymour ${ }^{\mathrm{a}}$, Rosalinda Inguanta ${ }^{\mathrm{b}}$, Alan O'Riordan ${ }^{\mathrm{a}}$

a Nanotechnology Group, Tyndall National Institute, University College Cork, Cork T12 R5CP, Ireland

b Laboratorio di Chimica Fisica Applicata, Dipartimento di Ingegneria, Università of Palermo, Viale delle Scienze, Palermo, Italy
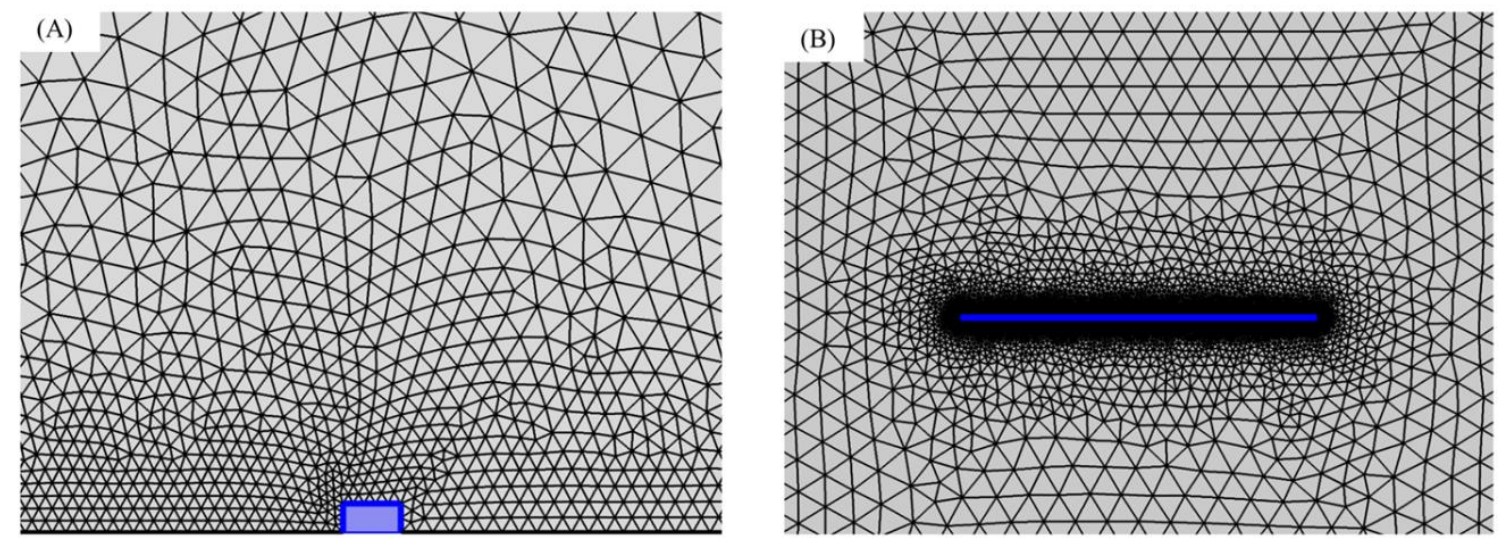

Figure S10 (A) Cross sectional view of 2D model meshing; (B) Plan view of 3D model meshing exhibiting higher mesh density. 
(A)

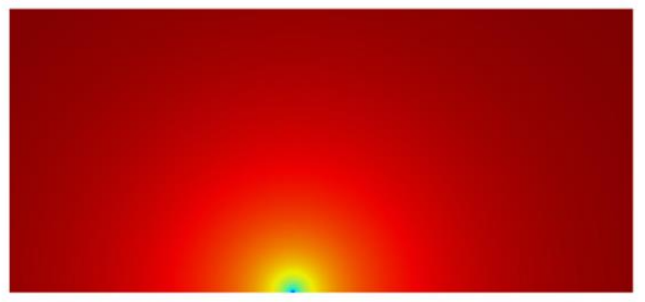

(C)

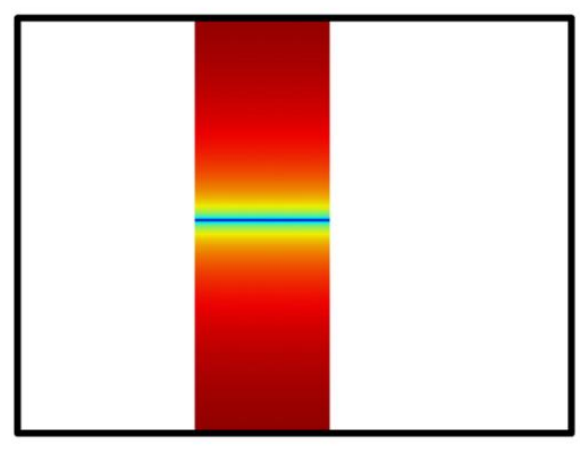

(B)

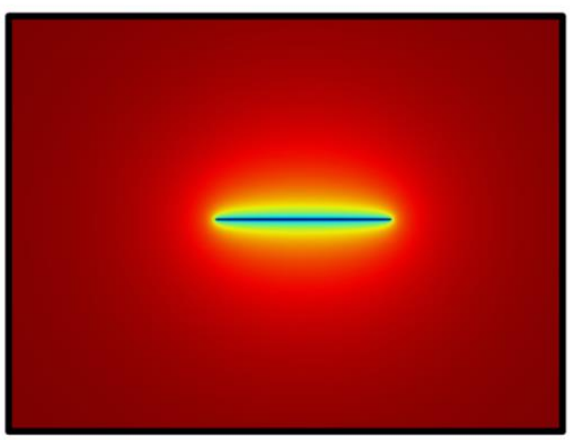

(D)

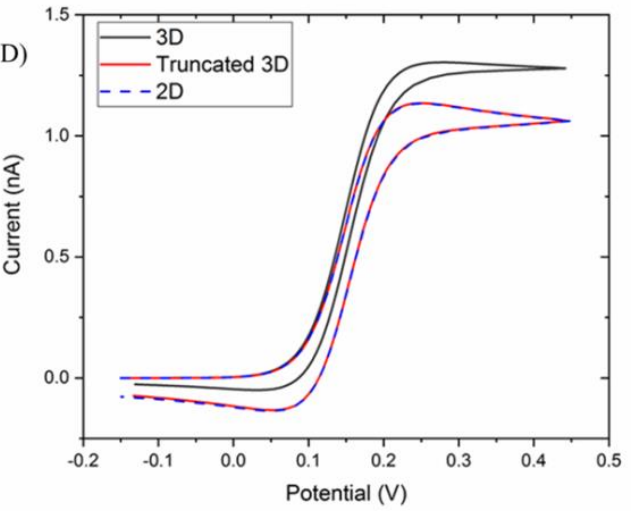

Figure S11 (A) Cross sectional view of 2D simulation of $\mathrm{FcCOOH}$ concentration profile for single nanowire (B) Plan view of 3D simulation of $1 \mathrm{mM}$ FcCOOH concentration profile for single nanowire (C) Plan view of 3D simulation with truncated domain of $1 \mathrm{mM} \mathrm{FcCOOH}$ concentration profile for single nanowire. (D) Cyclic voltammograms of single nanowire for 2D, 3D and truncated 3D models.

The accuracy of meshing is highly dependent on the level of meshing. Figure S2A and S2B show typical concentration profiles from a $2 \mathrm{D}$ and a plan view of the electrode from a $3 \mathrm{D}$ model. The diffusion to the electrode termini is proposed as the key source of error between the two models in terms of predicted current magnitude. In order to verify that there was no meshing contribution to the difference in current between the 2 models, the source was confirmed by design of a truncated 3D domain. The aim is to replicate solely what the DDA models but in 3D. The dimension of the domain in the $\mathrm{z}$-axis was set to that of the electrode length, with the domain walls set to zero flux. The resultant concentration profile is shown in Figure S2C. The diffusion profile is uniform the length of the electrode, with no edge effects possible due to the truncated domain. Figure S2D shows the simulated CVs of the 2D, 3D and truncated 3D model. It can be seen that the 3D CV overlaps that of the 2D model, confirming that the 3D differs from the 2D solely due to the diffusion from the electrode termini. 


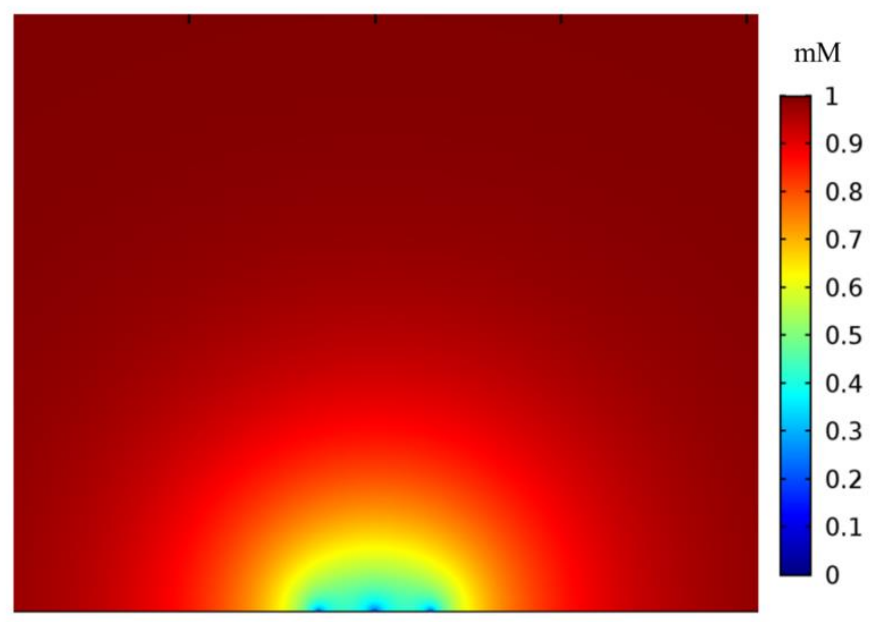

Figure S12 Cross sectional view of 2D simulation of $\mathrm{FcCOOH}$ concentration profile at array of 3 nanowires

(A)

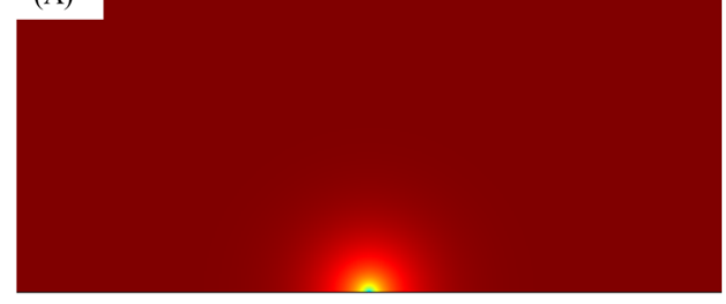

$\mathrm{mM}$

(B)

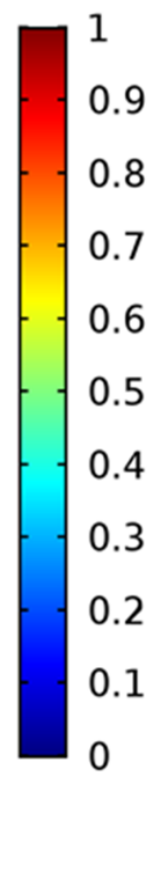

Figure S13 Cross sectional view of 2D simulation of $\mathrm{FcCOOH}$ concentration profile at array of 3 nanowires with an inter electrode gap of (A) $100 \mathrm{~nm}$ (B) 5 micron and (C) 200 micron; 

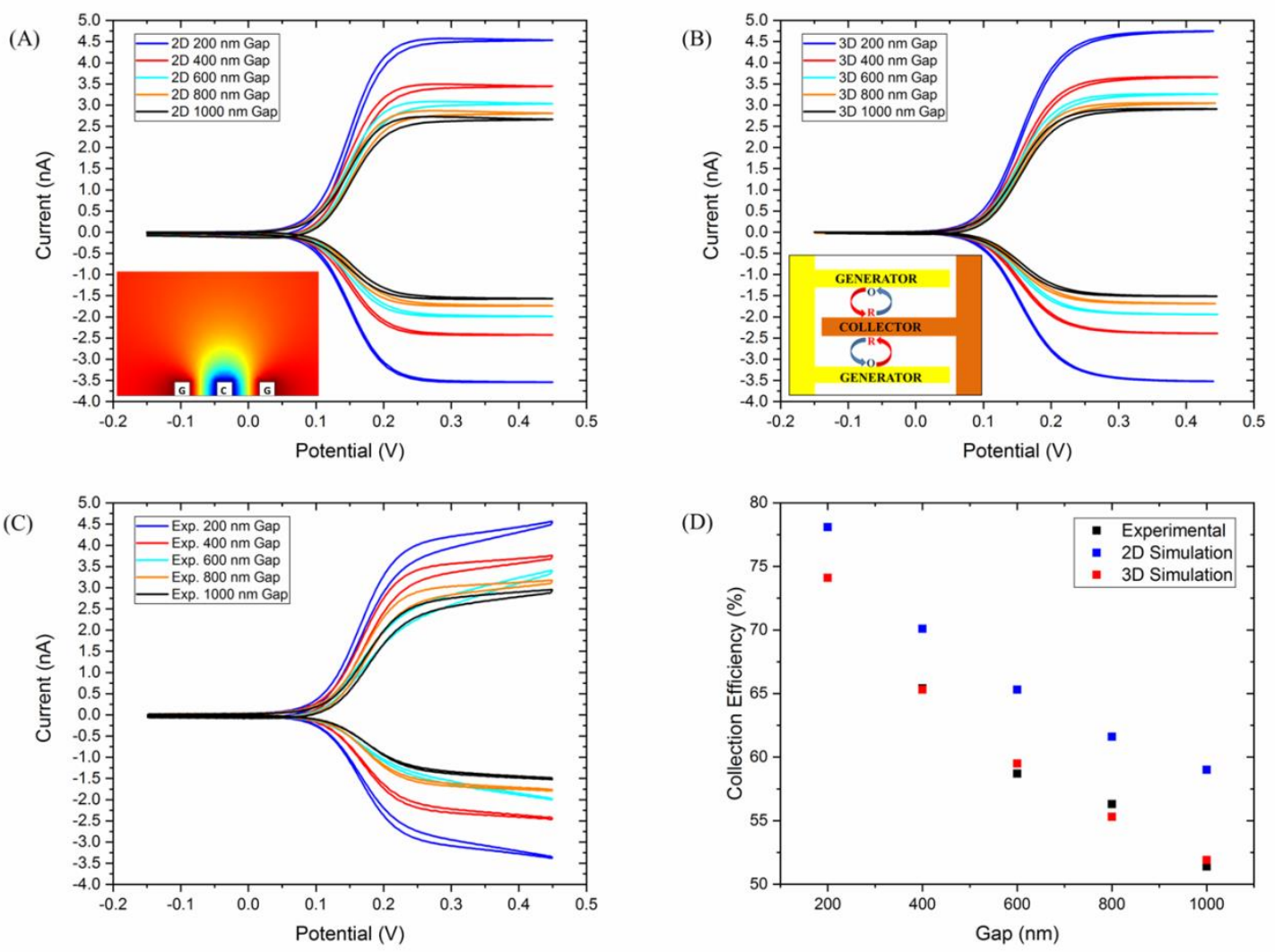

Figure S14 Cyclic voltammograms of $1 \mathrm{mM} \mathrm{FcCOOH}$ at interdigitated triplet electrode comprising of a single collector nanowire and two external generator nanowires in GC mode at a range of interelectrode gaps for (A) 2D simulations (inset: side-on concentration profile of $\mathrm{FcCOOH}$ at generator (G) and collector (C) electrodes), (B) 3D simulations (inset: schematic showing redox cycling between generators and collector), and (C) experimental data. The potential at the generators was cycled from -0.15 to $0.45 \mathrm{~V}$ while the collector were held at $-0.15 \mathrm{~V}$. (D) Plot of collection efficiency versus inter electrode gap from 2D simulation, 3D simulation and experimental current.

Further experimental validation of the generator collector simulations was performed by reversing the electrode set up used for Figure 8, now consisting of 2 generators and a single collector. Figure S5A shows the simulated currents for a pair of generators and a single collector nanowire electrode from a 2D model. Figure S5B shows the simulated currents for a pair of generators and a single collector nanowire electrode from a 3D model. Figure S5C shows the experimental data for a pair of generators and a single collector nanowire electrode. Figure S5D is a plot of collection efficiency for the current obtained from the 2D, 3D and experimental data. Collection efficiency again decreases with increasing electrode gap. The experimental data is again best described by the 3D model with the $2 \mathrm{D}$ overestimating the collection efficiency. The difference between the collection efficiencies is more significant for this single collector system, since all molecules reduced at the collector must pass by the generators ( $\mathrm{x}$ axis) by diffusion if they do not diffuse away up from the electrodes ( $\mathrm{z}$ axis). 

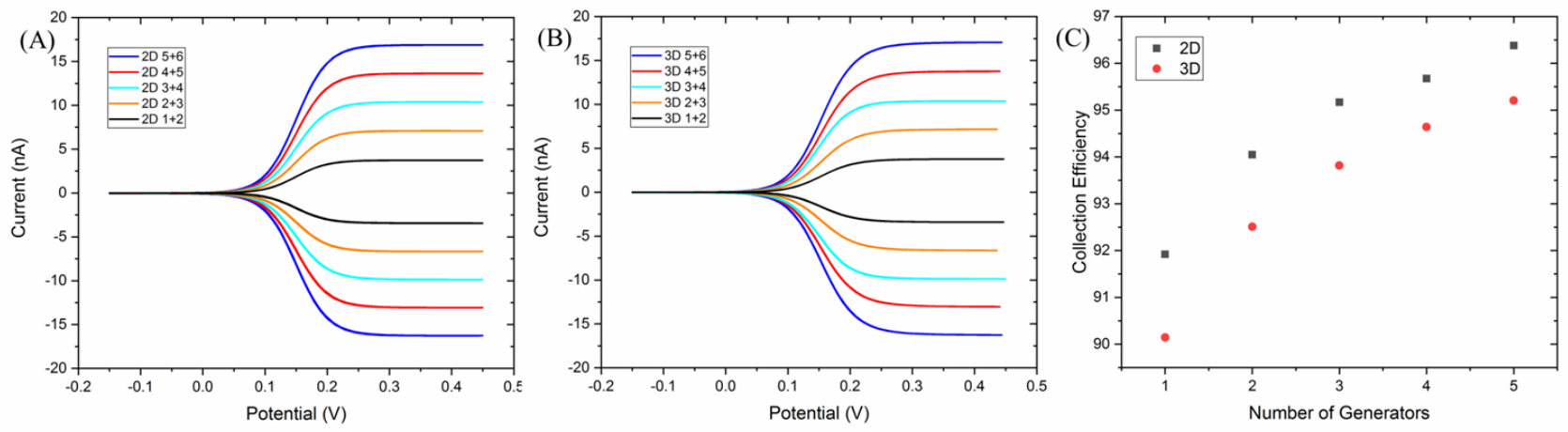

Figure S6 Theoretical cyclic voltammograms of $1 \mathrm{mM} \mathrm{FcCOOH}$ at $100 \mathrm{mV} / \mathrm{s}$ for $\mathrm{n}$ generator nanowires and $n+1$ collector nanowires at inter electrode gap of $500 \mathrm{~nm}$ for (A) 2D simulation and (B) 3D simulation; (C) Plot of collection efficiency veruss number of generator electrodes for 2D and 3D models.

Figure S6A shows the simulated currents from a 2D generator collector model of $\mathrm{n}$ generators and $n+1$ collectors with an inter-comb gap of $200 \mathrm{~nm}$. The current magnitude of both the generator and the collector combs increase with $\mathrm{n}$, as is expected with the increased total surface area with additional electrodes. Figure S6B presents the currents from a 3D model of the same electrode configurations. A similar trend to before is seen where the $2 \mathrm{D}$ generator current is lower but the collector current is higher than the 3D model. 